\title{
Short communication: Evaluation of amino acid consumption and necessary profiles of Streptococcus thermophilus T1C2 in controlled pH batch fermentations
}

\author{
C. Hong, Z. Shuang, X. Miao, Z. Min, L. Xin-Tong, D. Hong-Ling, M. Chun-Li, and F. Zhen ${ }^{1}$ \\ Key Laboratory of Dairy Science of Ministry of Education, College of Food Science, Northeast Agricultural University, 59 Mucai Road, \\ Harbin 150030, Heilongjiang, China
}

\section{ABSTRACT}

The objective of the present study was to elucidate the relationship between amino acid consumption and necessary profiles of Streptococcus thermophilus $\mathrm{T} 1 \mathrm{C} 2$ to guide the design of media for high-cell-density culture. The amino acid consumption and necessary patterns of $S$. thermophilus T1C2 were investigated in the complete chemically defined medium. For amino acid consumption profiles throughout the growth of $S$. thermophilus $\mathrm{T} 1 \mathrm{C} 2$, the most abundantly consumed amino acids were Gln and Arg, which accounted for 19 and $20 \%$ of total amino acids consumed, respectively. Asparagine, Thr, Ser, Ala, Val, Met, Leu, and Lys, consumptions of which ranged from 3 to $10 \%$ of total amino acids consumed, were the second most intensively consumed amino acids. For necessary amino acid patterns, the amount of Cys, which counted for $11 \%$ of total amino acids needed, was significantly higher than the amounts required for other amino acids in growth of $S$. thermophilus $\mathrm{T} 1 \mathrm{C} 2$. The necessary amounts of Asp, Asn, Glu, Gln, Arg, Ala, Met, and Tyr ranked second, ranging from 5 to $8 \%$ of total amino acids needed. Compared with necessary amounts, the consumption of Asn, Thr, Ser, Gln, Arg, Ala, Val, Leu, Lys, His, and Phe exceeded the necessary amounts for growth of $S$. thermophilus T1C2 remarkably. Consumption of Gly, Met, Ile, Trp, and Pro was slightly higher than the necessary amounts. Consumption of Asp, Glu, Tyr, and Cys was lower than the necessary amounts. The overall consumption of amino acids exceeded the required amount for growth of $S$. thermophilus T1C2 almost 2.43 times, which implied a significant nitrogen wasting.

Key words: Streptococcus thermophilus, amino acid, consumption, necessary

Received August 24, 2014.

Accepted January 10, 2015

${ }^{1}$ Corresponding author: NEAU_FengZhen@163.com

\section{Short Communication}

Streptococcus thermophilus, an essential lactic acid bacterium, has been commonly used for commercial production of yogurt and cheese. It is considered the second most important industrial dairy starter after Lactococcus lactis (Prajapati et al., 2013). Streptococcus thermophilus are fastidious organisms; they require not only carbohydrates as energy and carbon source, but also AA, nucleotides, and vitamins for growth in a defined medium. Their complex nutrient requirements are usually satisfied in natural or complex growth media by the addition of undefined compounds, such as peptone, meat, and yeast extract (Hongfei et al., 2013). The production of direct-vat-set yogurt is usually carried out in complex nutrient media to obtain relatively high biomass levels. Media design for high-cell-density cultivation is based on knowledge of nutritional requirements and necessary patterns for growth of $S$. thermophilus. However, current knowledge on nutritional requirements of $S$. thermophilus, especially AA consumption and requirement, is insufficient. The lack of reliable information on consumption and necessary patterns of AA hinders the rational design of cultivation media and optimization of bioprocesses.

For high-cell-density culture of S. thermophilus, protein hydrolysates, peptone, peptide, and yeast extract were common nitrogen sources for growth. However, current select tests of nitrogen sources do not consider the nutritional requirements of $S$. thermophilus. Furthermore, the proliferation effect of nitrogen source is dependent on AA consumption and necessary patterns of $S$. thermophilus. The media design of high-cell density cultivation therefore required thorough understanding of the AA requirements for $S$. thermophilus.

In a previous study, $S$. thermophilus $\mathrm{T} 1 \mathrm{C} 2$ was selected and displayed excellent properties in cheese and yogurt starter (Ma et al., 2011). The purpose of present study was to evaluate AA consumption and necessary patterns for growth of $S$. thermophilus $\mathrm{T} 1 \mathrm{C} 2$. The ultimate goal was to pave a way for high-cell density cultivation of $S$. thermophilus $\mathrm{T} 1 \mathrm{C} 2$. 
Streptococcus thermophilus T1C2 was cultivated from our previous study (Ma et al., 2011). Cultures were stored at $-70^{\circ} \mathrm{C}$ in $15 \%$ sterile reconstituted skim milk containing $10 \%$ glycerol. Complete chemically defined medium (CDM) was prepared as described in detail by Lahtvee et al. (2011). Batch experiments were performed in a 5-L Biotech-7000 bioreactor (Shanghai Baoxing, Shanghai, China). The reactor was filled with $1.5 \mathrm{~L}$ of culture medium. Temperature and rotation speed were fixed at $40^{\circ} \mathrm{C}$ and $80 \mathrm{rpm}$, respectively. The $\mathrm{pH}$ was maintained at 6.5 by automatic addition of 0.1 $\mathrm{mol} / \mathrm{L}$ of $\mathrm{NaOH}$.

Streptococcus thermophilus $\mathrm{T} 1 \mathrm{C} 2$ was precultured at $37^{\circ} \mathrm{C}$ in M17 broth for $16 \mathrm{~h}$. The culture was centrifuged $\left(10,000 \times g, 10 \mathrm{~min}, 4^{\circ} \mathrm{C}\right)$, and cells were washed twice with PBS buffer $(50 \mathrm{mmol} / \mathrm{L}, \mathrm{pH} 6.5)$. Inoculation of growth medium was carried out at $0.1 \%$ (vol/ vol); growth rate was measured by spectrophotometric measurement at $650 \mathrm{~nm}$. Biomass dry weight (DW) was determined gravimetrically. Each culture condition was repeated 5 times.

Free AA concentrations of supernatants were determined by an AA analyzer (Acquity UPLC; Waters Corp., Milford, MA). For measuring whole-cell AA composition of $S$. thermophilus $\mathrm{T} 1 \mathrm{C} 2$, biomass was hydrolysed with $6 \mathrm{~mol} / \mathrm{L}$ of $\mathrm{HCl}$ for $20 \mathrm{~h}$ at $120^{\circ} \mathrm{C}$. Tryptophan was measured by alkaline hydrolysis with $5 \mathrm{~mol} / \mathrm{L}$ of $\mathrm{NaOH}$ for $17 \mathrm{~h}$ at $100^{\circ} \mathrm{C}$. Determination of Cys was carried out according to method of Hoogerheide and Campbell (1992). Determination of Gln and Asn was performed according to methods of Kuhn et al. (1996) and Zhang et al. (2012). After hydrolyzing, AA were determined as free AA as described previously.

Based on AA concentrations in the cultivation broth, consumption patterns of the AA were calculated with average growth rate $(\boldsymbol{\mu}$; Figure 1$)$. The overall consumption of AA decreased from 13.82 to $11.09 \mathrm{mmol} / \mathrm{g}$ of DW with increasing $\mu$. The results indicated that the most abundantly consumed AA were Gln (19\% of total AA consumed) and Arg (20\% of total AA consumed) throughout the growth of $S$. thermophilus $\mathrm{T} 1 \mathrm{C} 2$, respectively, followed by the consumptions of Asn, Thr, Ser, Ala, Val, Met, Leu, and Lys, which ranged from 3 to $10 \%$ of total AA consumed; other AA had lower consumption amounts.

Based on AA concentrations in the whole-cell hydrolysate, necessary patterns of the AA were calculated with $\mu$ (Figure 2). The overall necessary amounts of AA had no significant change (from 5.23 to $5.03 \mathrm{mmol} / \mathrm{g}$ of DW) with increasing $\mu$. Cysteine was significantly high in necessary amounts of AA of S. thermophilus T1C2, which covered $11 \%$ of total AA needed, followed by necessary amounts of Asp, Asn, Glu, Gln, Arg, Ala, Met, and Tyr, which covered 5 to $8 \%$ of total AA needed; other AA had lower necessary amounts.

Compared with necessary amount, the consumption of Asn, Thr, Ser, Gln, Arg, Ala, Val, Leu, Lys, His, and Phe notably exceeded the necessary amounts for growth of S. thermophilus T1C2. Among which, Gln, Arg, Asn, Thr, Ser, Ala, Val, Leu, and Lys were intensively consumed, whereas the consumption amounts of His and Phe were relatively small. Serine, Gln, Arg, Val, Ala, Leu, Lys, His, and Phe were essential AA, whereas Asn and Thr were nonessential AA for $S$. thermophilus T1C2 (Figure 3). Furthermore, Gly, Met, Ile, Trp, and Pro were consumed slightly higher than the necessary amounts. Among which, Met was an intensively consumed AA, followed by the consumption amounts of Gly, Ile, Trp, and Pro. In addition, Met and Trp were essential AA, whereas Gly, Ile, and Pro were nonessential AA for S. thermophilus T1C2. Consumption of Asp, Glu, Tyr, and Cys was lower than needed for growth of $S$. thermophilus $\mathrm{T} 1 \mathrm{C} 2$; they were among the lowest consumption amounts of AA. In addition, Glu and Tyr were essential AA, whereas Asp and Cys were nonessential AA for $S$. thermophilus T1C2.

When 3 branched-chain AA were omitted from the complete CDM, Ile did not affect the growth of S. thermophilus $\mathrm{T} 1 \mathrm{C} 2$, whereas Val and Leu affected the growth of $S$. thermophilus T1C2 (Figure 3). This feature was in agreement with the observation for $S$. thermophilus ST18 in the complete CDM deprived of the branched-chain AA (Letort and Juillard, 2001). The results indicated that the biosynthesis of branchedchain AA might not meet requirements for growth of $S$. thermophilus $\mathrm{T} 1 \mathrm{C} 2$. In addition, growth of $S$. thermophilus $\mathrm{T} 1 \mathrm{C} 2$ was characterized by prolonged lag times (up to $12 \mathrm{~h}$ ) in a simplified growth medium containing essential AA (data not showed). This suggested that nonessential AA were also conducive to the growth of $S$. thermophilus $\mathrm{T} 1 \mathrm{C} 2$; similar results were also reported by Law and Kolstad (1983). Results of the present study indicated that essential AA did not have to have high amounts of consumption. The casein hydrolysates containing His, Lys, Glu, and Ser AA residua were beneficial for growth of $S$. thermophilus (Zhang et al., 2011). The soy protein hydrolysate, containing large amounts of Asp, Ile, Leu, His, Met, Glu, and Val, were able to promote the proliferation of $S$. thermophilus (Hongfei et al., 2013). According to the present study, it might be speculated that these protein hydrolysates contained AA that could meet AA consumption and necessary profiles of $S$. thermophilus. As His, Lys, Glu, Ser, Leu, His, Met, and Val were essential AA, Lys, Ser, Leu, Met, and Val were intensively consumed AA; Glu, Asp, and Met had high necessary amounts. 


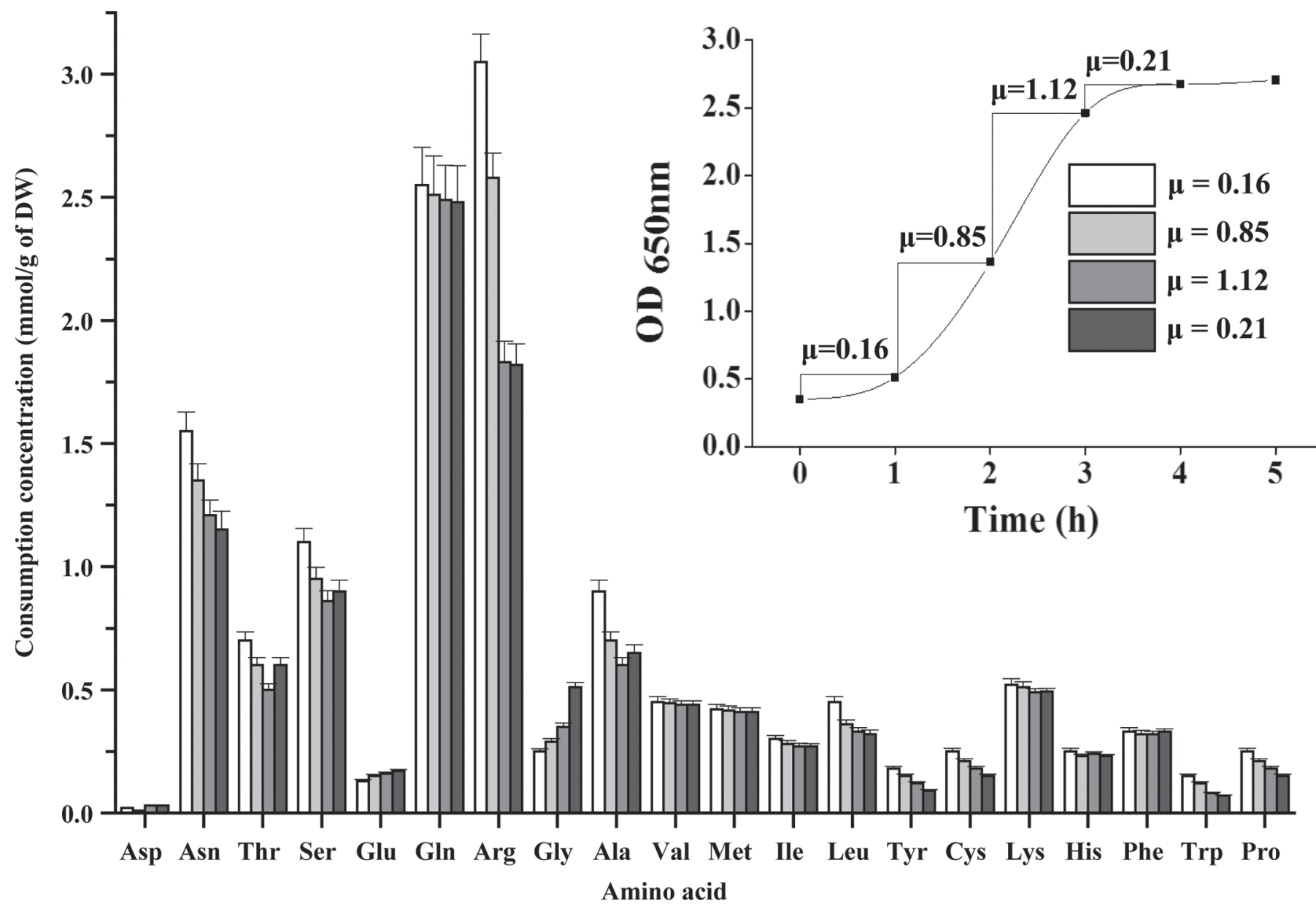

Figure 1. The AA consumption patterns of Streptococcus thermophilus $\mathrm{T} 1 \mathrm{C} 2$ at different average growth rates $(\mu)$. The columns represent the consumption concentrations $[\mathrm{mmol} / \mathrm{g}$ of dry weight (DW)] of AA at various average growth rates. Error bars represent SE.

Specifically, consumption of Arg decreased rapidly in the $\mu$ range of 0.16 to $1.12 / \mathrm{h}$ from $3.05 \mathrm{mmol} / \mathrm{g}$ of DW and levelled at $1.83 \mathrm{mmol} / \mathrm{g}$ of DW in the $\mu$ range of 1.12 to $0.21 / \mathrm{h}$. Consumption of Thr, Ser, and Ala decreased in the $\mu$ range of 0.16 to $1.12 / \mathrm{h}$ and increased in the $\mu$ range of 1.12 to $0.21 / \mathrm{h}$. Furthermore, necessary patterns of Thr, Ser, and Ala also showed the same trend. Consumption of Asn, Leu, Tyr, Pro, Cys, and Trp decreased, whereas Glu and Gly increased throughout the growth of S. thermophilus T1C2. Glycine was the only AA whose consumption increased throughout the growth of $S$. thermophilus $\mathrm{T} 1 \mathrm{C} 2$; however, its consumption was always lower than its need for biomass formation. Consumption of other AA did not change significantly throughout the studied $\mu$ range. These results may be attributed to factors including (1) different requirements of AA along with different $\mu$ of $S$. thermophilus $\mathrm{T} 1 \mathrm{C} 2$; (2) the consumption profiles of AA were partly dependent on necessary profiles of AA in $S$. thermophilus $\mathrm{T} 1 \mathrm{C} 2$; and (3) some AA may be converted to other substances.

The overall consumption of AA decreased from 13.82 to $11.09 \mathrm{mmol} / \mathrm{g}$ of DW with increasing $\mu$, indicating more efficient use of AA at higher $\mu$ values, as growth yields based on nitrogen consumption increased. As $\mu$ increased from 0.16 to $1.21 / \mathrm{h}$, biomass increased 1.9 times indicating a more efficient utilization of nitrogen for growth of $S$. thermophilus T1C2. Compared with necessary amounts (from 5.23 to $5.03 \mathrm{mmol} / \mathrm{g}$ of DW with increasing $\mu$ ), consumption of AA exceeded almost 2.43 times the required amounts for biomass production. Consumption of AA exceeded the necessary amounts for growth of $S$. thermophilus $\mathrm{T} 1 \mathrm{C} 2$, which implied that significant nitrogen wasting may be occurring. Similar results were reported by Lahtvee et al. (2011) in Lactococcus lactis. In the present study, nitrogen wasting may be mainly attributed to excessive consumption of Gln, Asn, and Arg. 


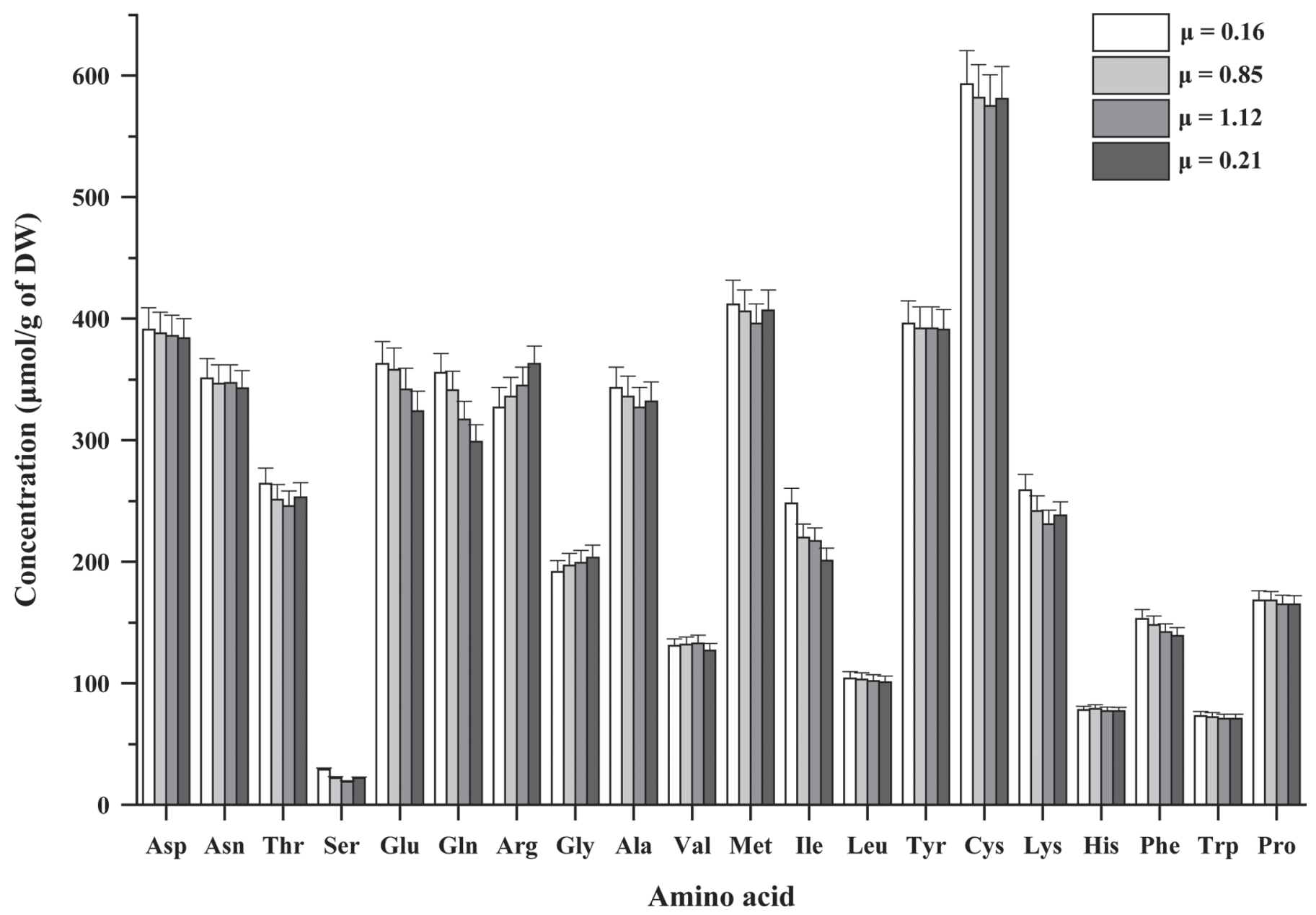

Figure 2. The necessary AA patterns of Streptococcus thermophilus $\mathrm{T} 1 \mathrm{C} 2$ at different average growth rates $(\mu)$. The columns represent the concentrations $[\mu \mathrm{mol} / \mathrm{g}$ of dry weight $(\mathrm{DW})]$ of AA at various average growth rates. Error bars represent SE.

The consumption of Gln, which was used for synthesis of proteins and the donor of amino groups in purine, pyrimidine, and in aminosugar production pathways, covered 18.5 and $22.5 \%$ of the total AA consumed at $\mu$ 0.16 and $1.21 / \mathrm{h}$, respectively. It could be assumed that maintenance of high intracellular concentrations of Gln was due to the fact that intense synthesis and consumption flows might be necessary to keep the transfer of AA effective (Lahtvee et al., 2011). Conversely, nitrogen wasting could be associated with the consumption of Asn, which was reduced from 1.55 to $1.21 \mathrm{mmol} / \mathrm{g}$ of DW with a $\mu$ increase from 0.16 to $1.21 / \mathrm{h}$. Asparagine and Asp were required for syntheses of nucleotides and proteins. In the present research, Asp was hardly consumed and therefore should have been produced from Asn. As with Gln, it could be assumed that overconsumption of Asn might be necessary to keep the transfer of amino group effective. Energetically, transport of Asn in L. lactis was much more efficient than
Asp (Benthin and Villadsen, 1996); moreover, Asn is probably preferentially directed toward the production of Asp (Konings et al., 1989; Lapujade at al., 1998). Surplus of Asp can be directed into pyruvate; Arg has dual effects on nitrogen wasting. With the increase of $\mu$ from 0.16 to $1.21 / \mathrm{h}$, biomass increased 1.9 times, showing that cells used nitrogen more effectively for $S$. thermophilus $\mathrm{T} 1 \mathrm{C} 2$. The most important AA was Arg, which played a role in the observed reduction of nitrogen wasting. Arginine consumption decreased from 3.05 to $1.83 \mathrm{mmol} / \mathrm{g}$ of DW with an increase of $\mu$ from 0.16 to $1.21 / \mathrm{h}$. Furthermore, Arg consumption was higher than necessary for $S$. thermophilus $\mathrm{T} 1 \mathrm{C} 2$, the majority of which may be transformed to ornithine (Figure 4), especially at lower specific growth rates, which indicated energy limitation of cells; similar results were reported for L. lactis (Lahtvee et al., 2011). However, not all Arg leftover from calculated requirements for biosynthesis was converted to ornithine. Although the mechanisms 


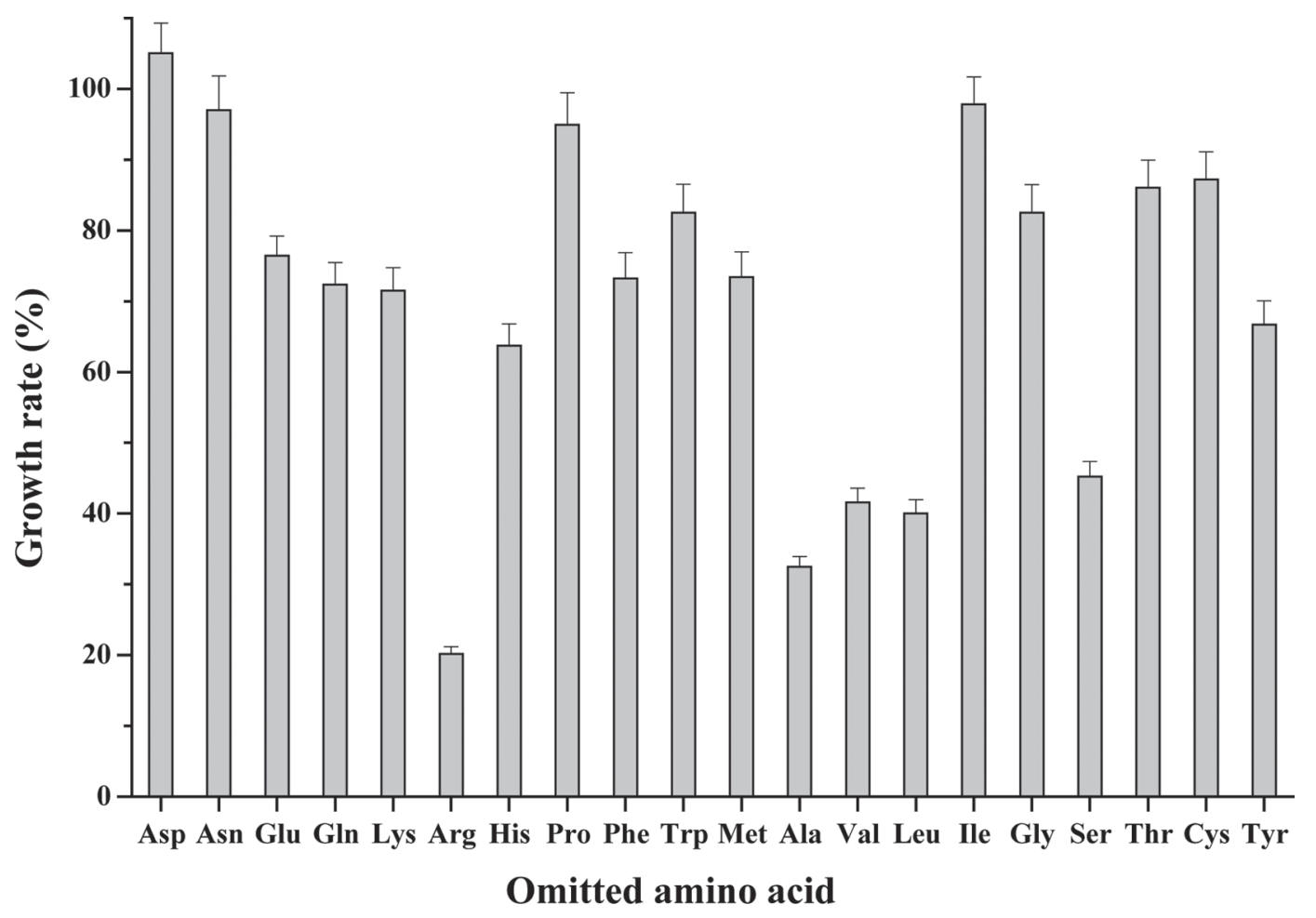

Figure 3. Effect of the omission of AA on the growth rate of Streptococcus thermophilus T1C2. The columns represent the growth rates of S. thermophilus $\mathrm{T} 1 \mathrm{C} 2$ when an AA was omitted from the chemically defined medium (CDM) compared with that in CDM with 20 AA. Growth rates were expressed as percentage of the growth rate in complete CDM. Error bars represent SE.

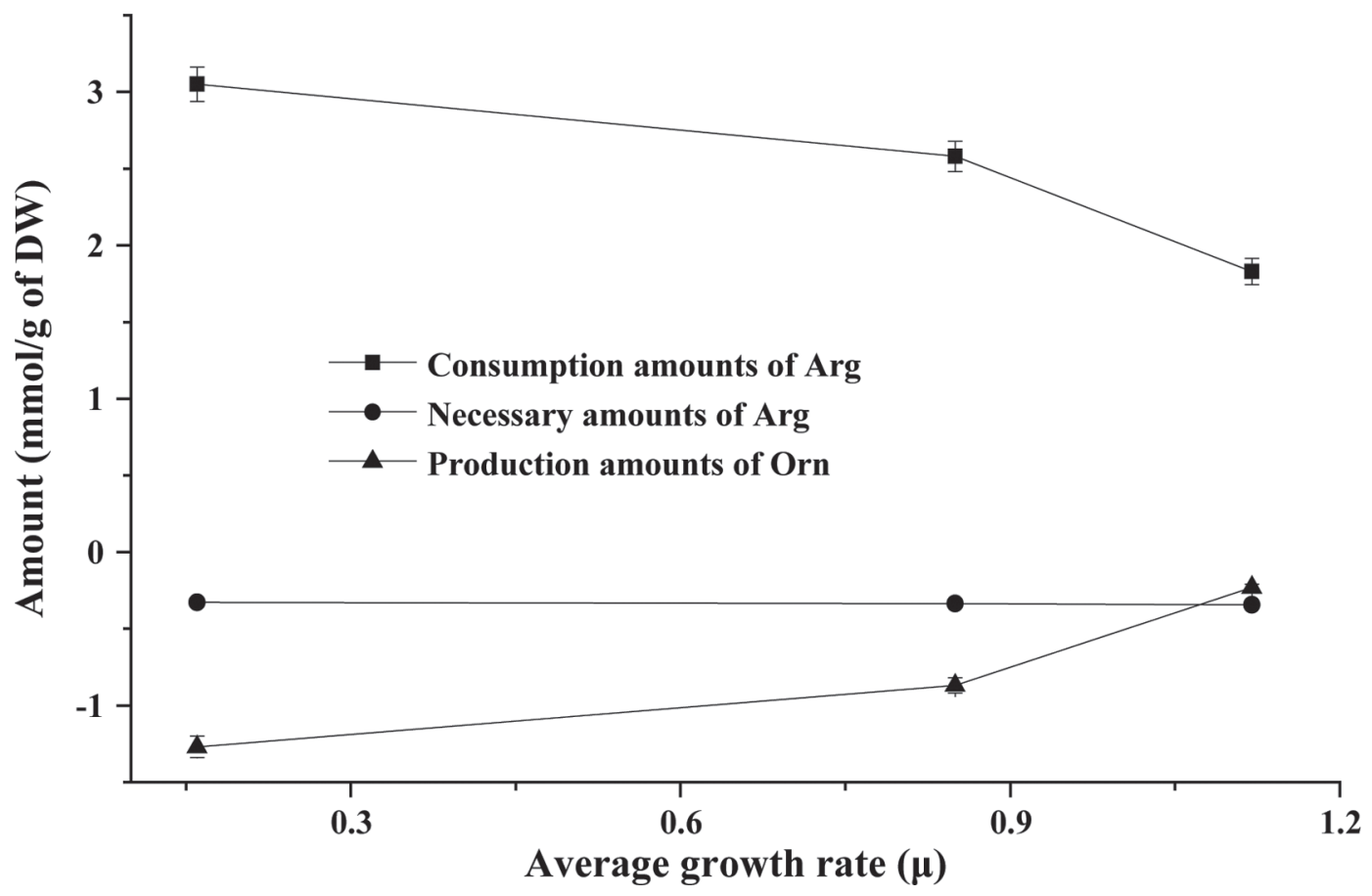

Figure 4. Consumption and necessary amounts of Arg and production of Orn in Streptococcus thermophilus T1C2. DW = dry weight. Error bars represent SE. 
of Arg overconsumption in addition to ornithine production are not known, production of Glu was observed when both Gln and Glu were present in the cultivation medium (Smid and Konings, 1990; Novak et al., 1997).

In terms of high-cell-density cultivation, distinct ratios of AA in the growth media were very important for biomass optimization. Exhaustion of an essential nutrient limits the growth of a culture by leading to a stationary growth phase. Thus, nutrient limitations could strongly hinder the achievement of desired results (Jan et al., 1999). As shown in our study, some AA were consumed in large amounts (Gln, Asn, and Arg) and efficient growth might not be achieved by insufficient supply of these compounds. Therefore, AA with large consumption and necessary amounts should be increased in the media, whereas those with low consumption and necessary amounts should be reduced. In addition, branched-chain and essential AA must also be considered. The consumption pattern, the necessary pattern of AA for S. thermophilus, which would be a better criterion for design media of high-cell-density cultivation, needs to be investigated in the future. In addition, how to reduce nitrogen wasting by designing rational ratios of $\mathrm{AA}$ in the growth media is still open to question.

In conclusion, our study suggested that the most abundantly consumed AA were Gln and Arg throughout the growth of $S$. thermophilus T1C2. Asparagine, Thr, Ser, Ala, Val, Met, Leu, and Lys were the next most intensively consumed AA. Cysteine was significantly higher than the necessary amounts of other AA for S. thermophilus $\mathrm{T} 1 \mathrm{C} 2$. The necessary amounts of Asp, Asn, Glu, Gln, Arg, Ala, Met, and Tyr ranked in the second place. Furthermore, consumption of Asn, Thr, Ser, Gln, Arg, Ala, Val, Leu, Lys, His, and Phe exceeded notably the necessary amounts for growth of S. thermophilus T1C2. Glycine, Met, Ile, Trp, and Pro were consumed in slightly higher amounts than needed for growth of S. thermophilus T1C2. Consumption of Asp, Glu, Tyr, and Cys was lower than needed for growth of $S$. thermophilus $\mathrm{T} 1 \mathrm{C} 2$. The overall consumption of AA exceeded the necessary amount for growth of S. thermophilus T1C2 almost 2.43 times, which implied significant nitrogen wasting. Overall, designing principles for properly balanced media and reducing nitrogen wasting by controlling ratios of $\mathrm{AA}$ in the growth media will be the focus of future research.

\section{REFERENCES}

Benthin, S., and J. Villadsen. 1996. Amino acid utilization by Lactococcus lactis ssp. cremoris FD1 during growth on yeast extract or casein peptone. J. Appl. Microbiol. 80:65-72.

Hongfei, Z., B. Fengling, Z. Fang, P. Walczak, J. Xiangning, and Z. Bolin. 2013. Characterization of soybean protein hydrolysates able to promote the proliferation of Streptococcus thermophilus ST. J. Food Sci. 78:M575-581.

Hoogerheide, J. G., and C. M. Campbell. 1992. Determination of cysteine plus half-cystine in protein and peptide hydrolysates: Use of dithiodiglycolic acid and phenylisothiocyanate derivatization. Anal. Biochem. 201:146-151.

Jan, W. S., V. Gerard, and K. Jan. 1999. Environmental stress responses in Lactococcus lactis. FEMS Microbiol. Rev. 23:483-501.

Konings, W. N., B. Poolman, A. J. M. Driessen, and P. C. Maloney. 1989. Bioenergetics and solute transport in lactococci. Crit. Rev. Microbiol. 16:419-476.

Kuhn, K. S., P. Stehle, and P. Fürst. 1996. Quantitative analyses of glutamine in peptides and proteins. J. Agric. Food Chem. 44:1808-1811.

Lahtvee, P.-J., K. Adamberg, L. Arike, R. Nahku, K. Aller, and R. Vilu. 2011. Multi-omics approach to study the growth efficiency and amino acid metabolism in Lactococcus lactis at various specific growth rates. Microb. Cell Fact. 10:12.

Lapujade, P., M. Cocaign-Bousquet, and P. Loubiere. 1998. Glutamate biosynthesis in Lactococcus lactis ssp. lactis NCDO 2118. Appl. Environ. Microbiol. 64:2485-2849.

Law, B. A., and J. Kolstad. 1983. Proteolytic systems in lactic acid bacteria. Antonie Van Leeuwenhoek 49:225-245.

Letort, C., and V. Juillard. 2001. Development of a minimal chemically-defined medium for the exponential growth of Streptococcus thermophilus. J. Appl. Microbiol. 91:1023-1029.

Ma, C. L., L. Zhang, H. Yi, M. Du, X. Han, L. Zhang, Z. Feng, Y. Zhang, and Q. Li. 2011. Technological characterization of lactococci isolated from traditional Chinese fermented milks. J. Dairy Sci. 94:1691-1696.

Novak, L., M. Cocaign-Bousquet, N. Lindley, and P. Loubiere. 1997. Metabolism and energetics of Lactococcus lactis during growth in complex or synthetic media. Appl. Environ. Microbiol. 63:26652670.

Prajapati, J. B., M. N. Neelam, K. P. Amrutlal, S. Suja, and G. J. Chaitanya. 2013. Genomic Analysis of Dairy Starter Culture Streptococcus thermophilus MTCC 5461. J. Microbiol. Biotechnol. 23:459-466.

Smid, E. J., and W. N. Konings. 1990. Relationship between utilization of Pro and Pro-containing peptides and growth of Lactococcus lactis. J. Bacteriol. 172:5286-5292.

Zhang, H., G. Tao, and H. Zhou. 2012. Quick determination of glutamine in soluble protein or peptide by UPLC. Sci. Technol. Food Indus. 33:338-365.

Zhang, Q., J. Ren, H. Zhao, M. Zhao, J. Xu, and Q. Zhao. 2011. Influence of casein hydrolysates on the growth and lactic acid production of Lactobacillus delbrueckii ssp. bulgaricus and Streptococcus thermophilus. Int. J. Food Sci. Technol. 46:1014-1020. 